\title{
Impact of Increasing Levels of Advanced Iterative Reconstruction on Image Quality in Low-Dose Cardiac CT Angiography
}

\section{Einfluss verschiedener Stufen iterativer Rekonstruktionstechnik auf die Bildqualität in der Niedrigdosis-CT-Angiografie der Koronargefäße}

Authors

Affiliations
P. Kröpil' , A. H. Bigdeli ${ }^{2}$, H. D. Nagel ${ }^{3}$, G. Antoch ${ }^{1}$, M. Cohnen ${ }^{2}$

Department of Diagnostic and Interventional Radiology, University Dusseldorf, Medical Faculty, Düsseldorf

Institute of Clinical Radiology, Städtische Kliniken Neuss Lukaskrankenhaus GmbH, Neuss

Science \& Technology for Radiology, Dr. HD Nagel, Buchholz
Key words

- cardiac CT

- iterative reconstruction

- image quality

- CT angiography

- dose

- iDose received 22.6.2013

accepted $\quad 16.10 .2013$

Bibliography

Dol http://dx.doi.org/

10.1055/s-0033-1356074

Published online: 23.1.2014

Fortschr Röntgenstr 2014; 186 :

567-575 @ Georg Thieme

Verlag KG Stuttgart · New York . ISSN 1438-9029

\section{Correspondence}

\section{Dr. Patric Kröpil}

Institut für Radiologie,

Universitätsklinikum Düsseldorf

Moorenstraße 5

40225 Düsseldorf

Germany

Tel.: ++49/211/8117754

Fax: $++49 / 211 / 8119487$

Patric.Kroepil@med.uni-

duesseldorf.de

\section{Zusammenfassung \\ $\nabla$}

Ziel: Untersuchung von Auswirkungen einer fortgeschrittenen iterativen Rekonstruktionstechnik (IR) auf die subjektive und objektive Bildqualität (IQ) in der Niedrigdosis CT-Coronarangiografie (CCTA).

Material und Methoden: 30 Datensätze prospektiv getriggerter „step-and-shoot“ CCTA wurden an einem 256-Schicht CT mit optimierten Dosisparametern akquiriert. Jeder Rohdatensatz wurde 16 mal an einem Prototyp für fortgeschrittene IR (iDose4, Philips) prozessiert, resultierend in $n=480$ Bilddatensätzen. Neben gefilterter Rückprojektion (FBP) wurden 4 Stufen zunehmender IR sowie zusätzlich zwei Faltungskerne ( $\mathrm{CB}$ und $\mathrm{XCB}$ ) und ein „multiresolution“ Algorithmus zur Bewahrung des Rauschleistungsspektrums verglichen. Kontrast-zuRausch Verhältnisse (CNR) wurden an 9 koronaren Lokalisationen bestimmt, die subjektive IQ auf einer 4-Punkt-Skala bewertet mit „klassischem“ Bildeindruck und rauschbedingten Artefakten als Hauptkriterien.

Ergebnisse: Bei einer effektiven Dosis von $1.7 \pm$ $0.7 \mathrm{mSv}$ wurde die mittlere CNR durch jede zunehmende IR-Stufe signifikant erhöht (Spanne $14.2-27.8 ; \mathrm{p}<0.001)$ mit bester objektiver IQ bei höchster IR-Stufe. Die subjektive IQ wurde bei mittlerer IR-Stufe (Level 4) am besten bewertet mit reduzierten rauschbedingten Artefakten und bewahrtem „klassischen“ Bildeindruck. Der kantenbetonende XCB-Faltungskern ergab bessere subjektive Bewertungen als der glättende CB-Kern ( $\mathrm{p}<0.05)$. „Multi-resolution“ führte zu einer weiteren Verbesserung der IQ bei hohen IR Stufen.

Schlussfolgerung: Die objektive IQ der Niedrigdosis CCTA verbessert sich mit steigendem Ausmaß iterativer Rekonstruktion. Die subjektive IQ ist hingegen bei mittlerer IR kombiniert mit kantenbetonendem XCB Kernel optimal durch ein Gleichge-

\section{Abstract \\ V}

Purpose: To investigate the effects of an advanced iterative reconstruction (IR) technique on subjective and objective image quality (IQ) in low-dose cardiac CT angiography (CCTA).

Materials and Methods: 30 datasets of prospectively triggered "step-and-shoot" CCTA scans acquired on a 256-slice CT scanner with optimized exposure settings were processed on a prototype IR system using filtered back-projection (FBP) and 4 levels of advanced IR (iDose4, Philips) providing incremental rates of IR (level 2, 4, 6, 7). In addition, the effects of different reconstruction kernels (semismooth [CB], standard with edge-enhancement [XCB]) and a "multi-resolution" feature [MR] to preserve the noise power spectrum were evaluated resulting in a total of $n=480$ image sets. Contrastto-noise ratios (CNR) were computed from regions of interest at 9 coronary locations. The subjective IQ was rated on a 4-point-scale with "classic" image appearance and noise-related artifacts as main criteria.

Results: At an effective dose of $1.7 \pm 0.7 \mathrm{mSv}$, the CNR significantly improved with every increasing level of IR (range: $14.2-27.8$; $\mathrm{p}<0.001$ ) with the best objective IQ at the highest level of IR (level 7). The subjective IQ however, was rated best at the medium level of IR (level 4) with minimal artifacts and a more "classic" image appearance when compared to higher IR levels. The XCB kernel provided better subjective ratings than $\mathrm{CB}$ $(p<0.05)$ and the MR feature further increased the IQ at a high level of IR.

Conclusion: The objective IQ of low-dose CCTA progressively improves with an increasing level of IR. The best subjective IQ however, is reached at medium levels of IR combined with an edgeenhancing kernel allowing for preservation of a "classic" image appearance suggesting application in the clinical routine. 
wicht von Rauschreduktion und Erhalt des „klassischen“ Bildeindrucks.

Kernaussagen:

- iterative Rekonstruktionstechnik verbessert die Bildqualität der Niedrigdosis CT-Coronarangiografie

- die objektive Bildqualität (CNR) steigt mit dem Ausmaß iterativer Rekonstruktion

- optimale subjektive Bildqualität wird bei mittlerer Stufe iterativer Rekonstruktion erreicht

> der „multi-resolution“ Algorithmus verbessert zusätzlich die Bildqualität bei hoher Stufe iterativer Rekonstruktion
Key Points:

- iterative reconstruction (IR) improves image quality in lowdose coronary CTA

- objective image quality (CNR) enhances with increasing level of IR

- best subjective image quality is reached at medium level of IR

- „multi-resolution“ algorithm further improves image quality at a higher level of IR

Citation Format:

- Kröpil P, Bigdeli AH, Nagel HD etal. Impact of Increasing Levels of Advanced Iterative Reconstruction on Image Quality in Low-Dose Cardiac CT Angiography. Fortschr Röntgenstr 2014; 186: 567-575

\section{Introduction \\ $\nabla$}

Cardiac computed tomography angiography (CCTA) enables reliable noninvasive examination of the coronary arteries. Improvements in scanner technology since the 1990 s have resulted in excellent temporal and spatial resolution with adequate visualization of up to $97 \%$ of coronary segments [1, 2]. Coronary artery disease (CAD) can now be excluded by CCTA with a high negative predictive value, thus helping to avoid unnecessary invasive angiography $[3,4]$. CCTA initially required radiation doses of up to $30 \mathrm{mSv}$ due to retrospective ECG gating combined with a very low pitch and the challenge of visualizing small vessels [5]. Therefore, radiation exposure of CCTA has become a major concern in clinical practice [6], and increasing awareness developed among the medical community. Many innovations regarding radiation protection in CCTA including a tailored approach concerning tube potential (low $\mathrm{kV}$ techniques) [7], ECGbased tube current modulation [8], and prospectively ECG-triggered image acquisition ("step-and-shoot") have been consecutively introduced. Using the "step-and-shoot" technique, mean radiation doses of less than $5 \mathrm{mSv}$ are achievable [9-11]. However, image quality remains an essential parameter for all lowdose examinations as accurate visualization of the coronary tree is critical for CCTA.

Filtered back-projection (FBP) has been the preferred method of CT image reconstruction since the $1970 \mathrm{~s}$ due to its fast and robust results [12]. Recently, CT reconstruction techniques, which are based on iterative reconstruction (IR) algorithms, have become available for clinical CT. IR techniques use correction loops to progressively refine image data and to separate image information from noise. They offer a potential radiation dose reduction while maintaining high image quality [13-15]. With early IR algorithms, effective noise reduction could be achieved by processing data in image space similar to adaptive postprocessing filters [16-18]. Advanced IR systems also involve the raw data space and are even more powerful with respect to reducing and preventing artifacts by integrating anatomy-based model calculations.

This study was done to investigate the effects of an advanced 4th generation IR technique on subjective and objective image quality (IQ) in low-dose cardiac CT angiography (CCTA).

\section{Materials and Methods \\ V}

Patients

In this retrospective study we analyzed 30 contrast-enhanced prospectively ECG-triggered CCTA examinations in 30 consecutive patients performed in a dose-optimized "step-and-shoot" scan protocol between July and August 2011. Study patients (15 female, 15 male; mean age: $61.3 \pm 12.9$ years) were all referred for CCTA from the department of cardiology. The mean BMI was $25.4 \pm 4$.6. Indications for CCTA were high or intermediate pretest probability for $\operatorname{CAD}(\mathrm{n}=17)$, known CAD with clinical aggravation (no evidence of acute myocardial infarction) $(n=11)$, typical or atypical chest pain $(n=4)$, and evaluation of bypass grafts $(n=3)$. A heart rate of $60 \mathrm{bpm}$ or lower and a sinus rhythm were targeted. If necessary, the patient's heart rate was controlled with intravenous (i. v.) beta-blocker (metoprolol $5 \mathrm{mg}$, Beloc ${ }^{\circledR}$ AstraZeneca, Wedel, Germany) immediately before the scan. Patients with an irregular rhythm or heart rate higher than $70 \mathrm{bpm}$ were excluded from "step-and-shoot" mode scanning and were not included in the study. All patients received nitroglycerine (Nitrolingual akut ${ }^{\circledR} 0.4 \mathrm{mg}$, Pohl-Boskamp, Hohenlockstedt, Germany) sublingually prior to CCTA.

\section{MDCT scan protocol}

CCTA was performed on a 256-row CT scanner (Brilliance iCT, Philips, Best, Netherlands). The imaging protocol included anterior-posterior scout images and a non-contrast scan to assess the coronary calcium score if clinically indicated. CCTA was obtained after the administration of contrast agent (iomeprol, Imeron $350^{\mathrm{TM}}$, Bracco Imaging Group, Italy). The amount of contrast and the flow rate were adapted to body weight in accordance with $\bullet$ Table 1. Injection of contrast was followed by a $40 \mathrm{ml}$ saline flush at the same flow rate. The start time of CCTA data acquisition was determined by a computer-assisted bolus tracking program with a trigger threshold of $120 \mathrm{HU}$ in the descending aorta; CT data acquisition was started $12 \mathrm{~s}$ after triggering. The scan parameters included a $128 \times 0.625 \mathrm{~mm}$ collimation, gantry rotation time of $270 \mathrm{~ms}$, tube potential of $100-120 \mathrm{kV}$, and a tube load of between 100 and 200 electrical mAs. The tube potential and tube load were selected according to the patient's chest size ( $\bullet$ Table 2 ). The $\mathrm{z}$-axis field of view extended from the carina or pulmonary artery segment down to the diaphragm for native CCTA. In patients with coronary artery bypass graft surgery, the z-coverage was extended. ECG-triggered image acquisition started at $78 \%$ of the RR interval. Axial images were reconstructed with a slice thickness of $0.6 \mathrm{~mm}$ at a reconstruction increment of $0.5 \mathrm{~mm}$. 
Table 1 The volume and flow rate of the contrast medium was adapted to the body weight of the patients.

Tab. 1 Volumen und Flussrate des Kontrastmittels wurde an das Körpergewicht des Patienten angepasst, um eine konstante Kontrastierung zu erreichen.

\begin{tabular}{|llll|}
$\begin{array}{l}\text { body weight } \\
\text { [kg] }\end{array}$ & $\begin{array}{l}\text { contrast volume } \\
\text { [ml] }\end{array}$ & flow rate $[\mathrm{ml} / \mathbf{s}]$ & $\begin{array}{l}\text { number of } \\
\text { patients }\end{array}$ \\
\hline$<90$ & 70 & 5 & 23 \\
\hline 90 to 100 & 80 & 5.5 & 5 \\
\hline 100 to 110 & 90 & 6 & 2 \\
\hline
\end{tabular}

Table 2 Mean values \pm standard deviation for dose-related parameters in the patients of the study group.

Tab. 2 Mittelwerte \pm Standardabweichung der dosisrelevanten Parameter der Studienpatienten

\begin{tabular}{|lr|}
\hline tube potential $120 \mathrm{kVp}, \mathrm{n}$ & 5 \\
\hline tube potential $100 \mathrm{kVp}, \mathrm{n}$ & 25 \\
\hline tube current time product, mean $\pm \mathrm{SD}[\mathrm{mAs}]$ & $172 \pm 39$ \\
\hline CTDIvol, mean \pm SD [mGy] & $9.8 \pm 3.8$ \\
\hline dose length product $(\mathrm{DLP})$, mean \pm SD [mGy $\cdot \mathrm{cm}]$ & $124 \pm 46$ \\
\hline scan length, mean \pm SD [cm] & $12.7 \pm 0.6$ \\
\hline
\end{tabular}

\section{Image reconstruction (processing)}

Data were transferred to and processed on a dedicated separate prototype $4^{\text {th }}$ generation iterative reconstruction (IR) system (iDose ${ }^{4}$, Philips Healthcare, Netherlands). This system is a hybrid IR algorithm that operates in both the raw data and the image data domain, thereby reducing streak artifacts caused by photon starvation and image noise. It offers seven different level settings, defining the strength of the IR algorithm. Increasing iDose ${ }^{4}$ levels indicate an increasing strength of noise reduction (range: 11 $55 \%$ noise reduction relative to a corresponding FBP reconstruction). The level can be defined independently from the radiation dose at which an acquisition is performed. In addition, iDose ${ }^{4}$ provides a feature named "multi-resolution", which is a multifrequency noise removal technique that lowers the overall noise while closely preserving the desired frequency spectrum characteristic of a corresponding routine-dose FBP image to preserve the image texture and maintain an image appearance that is familiar to clinicians [19].

The raw dataset of each CCTA was processed 16 times resulting in $\mathrm{n}=30$ patients $\times 16$ reconstructions $=480$ image datasets using filtered back-projection (FBP) and 4 levels of an advanced IR technique providing an incremental rate of IR (iDose level 2, 4, 6 and 7). For higher levels of IR (iDose level 4 and above), processing was performed both without and with a "multi-resolution" feature. In addition, two different cardiac reconstruction kernels (CB: cardiac standard; XCB: cardiac standard with edge enhancement) were used with FBP and each IR level, respectively. Both kernels are used for cardiac imaging. By using an XCB kernel, additional vessel wall enhancement can be achieved. Axial $0.8 \mathrm{~mm}$ images were reconstructed as well as straight and curved multiplanar reformations (MPR), and a $3 \mathrm{D}$ reconstruction in volume rendering technique (VRT), respectively.

\section{Image analysis}

The objective image quality was evaluated in all 30 datasets as previously described in detail [2]. The following measurements were obtained by one reader (A.H.B.) using $0.8 \mathrm{~mm}$ thick axial images.
Circular regions of interest (as large as possible, $2-4 \mathrm{~mm}^{2}$ ) were drawn in the lumen of the coronary arteries and the adjacent epicardial fatty tissue in order to derive the contrast-to-noise ratio (CNR) from the corresponding CT numbers in nine locations: left main coronary artery (LM), proximal and distal (distal to the second diagonal branch) left anterior descending coronary artery (LAD), proximal first diagonal branch (D1), proximal and distal left circumflex coronary artery (LCX), first obtuse marginal branch (OM1), proximal and distal (proximal to the origin of the posterior descending coronary artery) right coronary artery (RCA).

A circular region of interest $\left(100 \mathrm{~mm}^{2}\right)$ was placed in the contrastenhanced lumen of the aortic root to measure the image noise by determining the standard deviation of $\mathrm{CT}$ numbers $[20,21]$. The contrast-to-noise ratio (CNR) was determined for all 9 coronary locations by the following formula as described previously [2]: contrast-to-noise ratio $=(\mathrm{CT}$ number coronary lumen $[\mathrm{HU}]-\mathrm{CT}$ number adjacent tissue [HU]) / image noise [HU].

In addition, datasets of 11 patients $(n=4$ without coronary abnormalities, $n=3$ with coronary stenosis, $n=2$ with coronary stents, $\mathrm{n}=2$ with bypass grafts) were transferred to an offline workstation (Brilliance Workspace 4.5, Philips, Best, Netherlands). The subjective image quality was evaluated in all 16 different reconstructions of these datasets by three independent readers (M.C., P.K., A.H.B.) with different levels of experience in cardiac imaging (10, 7 and 2 years, respectively). The readers were blinded to the level of IR. Based on the 18-segment model of the Society of Cardiovascular Computed Tomography, subjective scores were given for every segment using a four-point scale ( 1 =excellent image quality: "classic" image appearance, no or minimal artifacts; 2 = good image quality: slight artifacts, artificial image appearance; 3 = moderate image quality: artifacts mainly due to noise, but evaluable concerning the presence of stenosis; $4=$ unevaluable). The main criteria of subjective image quality were "classic" image appearance (CT image appearance as known by and familiar to radiologists) and artifacts due to noise.

\section{Radiation dose}

The volume CT dose index (CTDIvol) and dose-length product (DLP) were obtained for all scans using the dose exposure record generated by the scanner. Additionally, the patient's effective dose (mSv) according to ICRP 60 was estimated using the DLP method with a conversion factor $\mathrm{k}=0.014 \mathrm{mSv} / \mathrm{mGy} \times \mathrm{cm}[22]$.

\section{Statistical analysis}

The statistical analysis was performed using commercially available software (SPSS, 20.0, Inc., Chicago, IL, USA; Microsoft Excel, Redmond, WA, USA). Continuous data are expressed as mean \pm SD. Differences of CNR and subjective image quality among different levels of IR and different reconstruction kernels were examined using one-way analysis of variance (ANOVA). A two-tailed pvalue $<0.05$ was considered statistically significant. Fleiss kappa with correction according to Brennan and Prediger was determined for interobserver agreement of subjective image quality. The coefficient represents concordance, where 1 is perfect agreement and 0 is no agreement at all.

\section{Results}

Patient characteristics

The mean heart rate during the scan was $56.5 \pm 5.7 \mathrm{bpm}$ (range $45-68 \mathrm{bpm}$ ). A dose of $10.0 \mathrm{mg}$ i. v. metoprolol was administered 
to two (6.7\%) of the patients. A mean flow rate of $5.1 \pm 0.2 \mathrm{ml} / \mathrm{s}$ for a mean amount of $73 \pm 6 \mathrm{ml}$ of contrast agent was injected.

\section{Radiation dose}

The dose-related parameters of the 30 CCTA scans in this study are listed in Table 2 . The mean z-axis scan length was $12.7 \pm$ $6.2 \mathrm{~cm}$. The mean DLP for the 30 single prospectively triggered CCTA acquisitions was $124 \pm 46 \mathrm{mGy} \times \mathrm{cm}$ corresponding to an estimated effective dose of $1.7 \pm 0.7 \mathrm{mSv}$ (range $0.9-3.0$; conversion factor $0.014 \mathrm{mSv} / \mathrm{mGy} \times \mathrm{cm}$ ). Patients with a BMI <25 demonstrated a mean effective dose of $1.2 \pm 0.4 \mathrm{mSv}$ for CCTA (range $0.9-2.1)$.

\section{Objective image quality}

The average CT numbers were as follows: $520 \pm 121 \mathrm{HU}$ (aortic root, range 312 to $796 \mathrm{HU}$ ), $432 \pm 123 \mathrm{HU}$ (coronaries, range 130 to $838 \mathrm{HU}$ ) and $-91 \pm 15 \mathrm{HU}$ (adjacent epicardial fatty tissue, range -19 to $-146 \mathrm{HU}$ ). While the CT numbers in the aortic root (ROI size: $100 \mathrm{~mm}^{2}$ ) remained constant (within $\pm 1 \mathrm{HU}$ ), the corresponding CT numbers in the coronaries (ROI size: $1-2 \mathrm{~mm}^{2}$ ) were somewhat shifted towards lower values with increasing iDose levels: by -10 and -4 on average for kernel $\mathrm{CB}$ (w/o and with multi-resolution, respectively), and by -19 and -7 on average for kernel XCB (w/o and with "multi-resolution", respectively).

The mean CNR of all measured locations in all reconstructions was 21.5 \pm 5.3 . The mean CNR of all nine examined regions of the coronary arteries with respect to FBP and the different levels of IR are shown for the $\mathrm{CB}$ kernel in 0 Fig. $1 \mathrm{~A}$ and for the XCB kernel in $\odot$ Fig. 1B. The mean CNR was significantly improved with IR when compared to FBP and with every increasing level of IR (range CNR: 14.2 - 27.8; $\mathrm{p}<0.001$ ) with the best objective IQ at the highest level of IR (iDose level 7) ( $\bullet$ Fig. 1). When using the CB kernel, the CNR was significantly higher compared to the XCB kernel at FBP and iDose level 2, 4 and 6. At iDose level 7 the differences failed statistical significance ( $\bullet$ Fig. 2). At the highest level of IR (iDose level 7), the "multi-resolution" feature led to a further significant improvement in the CNR (32.9; $\mathrm{p}<0.001$ ) ( $\bullet$ Fig. 3).

\section{Subjective image analysis}

In 11 patients coronary artery segments were analyzed for subjective image quality. Each of these segments was scored in all 16 reconstructions resulting in 5829 scores. An image quality score of 1 ("excellent") was given 1024 times (17.6\%), a score of 2 ("good") 2933 times (50.3\%), a score of 3 ("moderate") 1747 times (30.0\%), and 4 "unevaluable" was scored 125 times (2.1\%). The predominant reasons for "unevaluable" segments given by the observers were motion, noise, or streak artifacts due to extensive calcifications.

When using the $\mathrm{CB}$ kernel, the mean rating score over all patients and segments was $2.4 \pm 0.5$ for FBP, $2.3 \pm 0.6$ for iDose level 2, $2.1 \pm 0.7$ for iDose level 4, $2.4 \pm 0.6$ for iDose level 6 and $2.8 \pm 0.6$ for iDose level 7, respectively ( $\bullet$ Fig. 4A). When using the XCB kernel, the mean rating score for all patients and segments was $2.3 \pm 0.5$ for FBP, $2.0 \pm 0.7$ for iDose level 2, 1.7 \pm 0.7 for iDose level 4, $2.1 \pm 0.5$ for iDose level 6 and $2.6 \pm 0.6$ for iDose level 7, respectively ( $\bullet$ Fig. 4B).
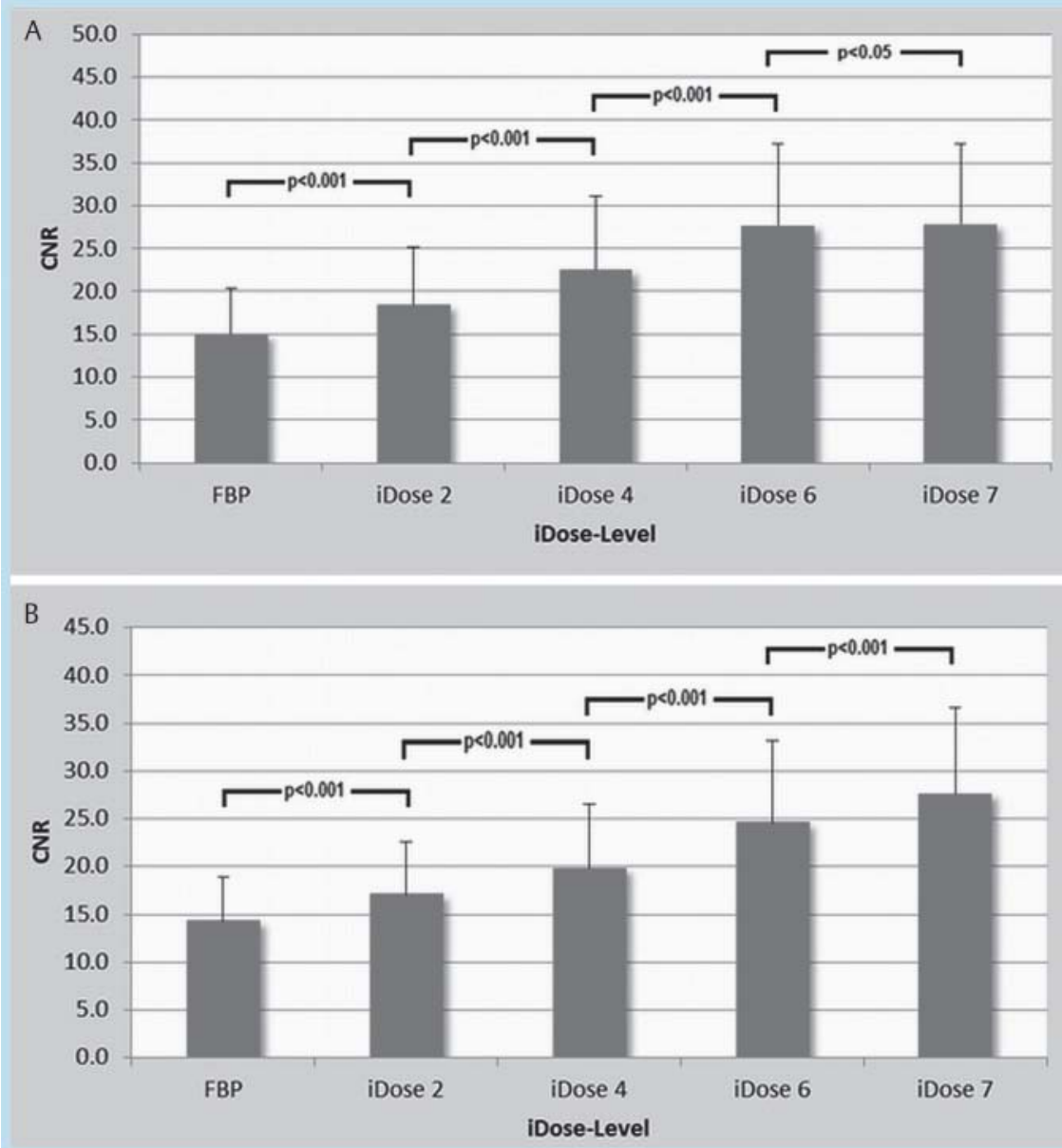

Fig. 1 CNR values at different levels of IR. Significant improvement of CNR was found for IR when compared to FBP and for every increasing level of IR when using CB kernel (A) or XCB kernel (B).

Abb. 1 CNR-Werte bei unterschiedlichen IR-Stufen. Signifikante Verbesserung des CNR bei IR im Vergleich zur FBP und für jede aufsteigende IR-Stufe unter Verwendung eines CB-Faltungskerns ( $(A)$ oder XCB-Faltungskerns (B). 

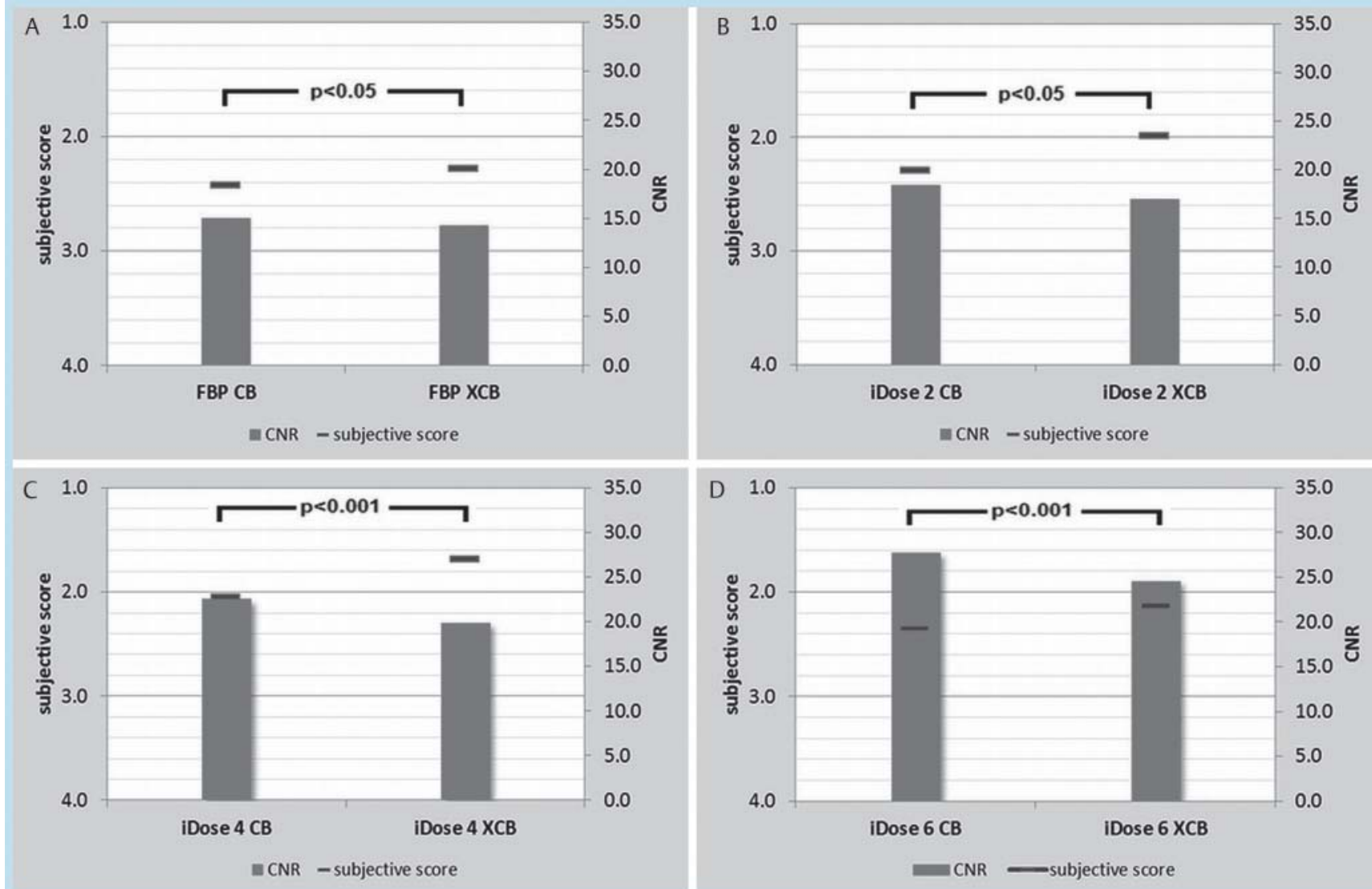

Fig. 2 Comparison of CNR and subjective image quality for CB kernel and XCB kernel, respectively. The CNR was significantly better for $C B$ kernel at FBP (A), iDose level 2 (B), level 4 (C) and level 6 (D) as compared to XCB kernel. With respect to the subjective scores, however, the XCB kernel was superior to the CB kernel (A-D).

Abb.2 Vergleich der CNR und der subjektiven Bildqualität für den CB und XCB-Faltungskern. CNR war significant höher für CB bei FBP (A), iDose Stufe 2 (B), 4 (C) und 6 (D) im Vergleich zum XCB-Faltungskern. Hinsichtlich der subjektiven Bildqualität war allerdings der XC-Faltungskern überlegen (A-D).

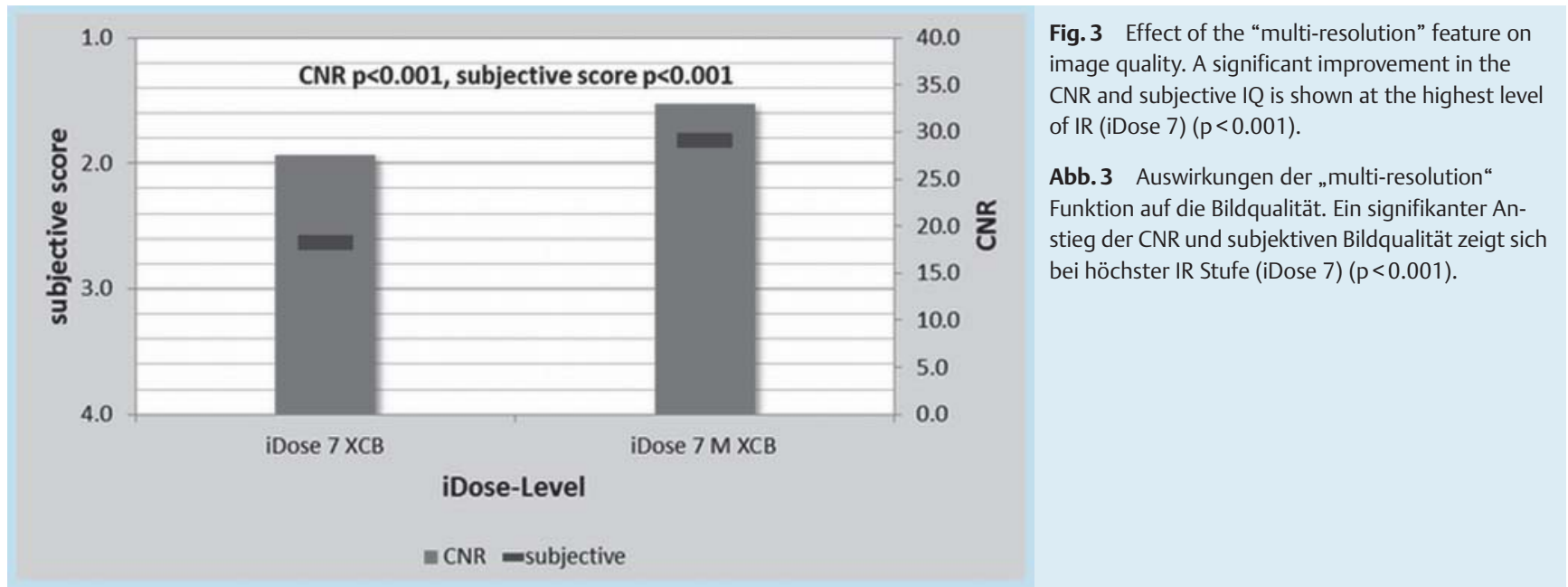

The subjective IQ was rated best at a medium level of IR (iDose level 4) with significant improvement for IR when compared to FBP $(p<0.0001)$ and a more "classic" appearance of images compared to high levels of IR $(p<0.001)(\bullet$ Fig. 4, 5). For FBP and each level of IR, the subjective IQ score was better when using the XCB kernel when compared to the $\mathrm{CB}$ kernel reconstructions (० Fig. 2).
When using the "multi-resolution" feature and CB kernel, the mean score for all patients and segments was $1.7 \pm 0.6$ for iDose level 4, $2.6 \pm 0.6$ for iDose level 6 and $2.9 \pm 0.5$ for iDose level 7, respectively, showing a significant improvement at iDose level 4 ( $p<0.001$ ) only. When combining the multi-resolution feature and the XCB kernel, the mean rating score for all patients and segments was $1.4 \pm 0.5$ for iDose level $4,1.6 \pm 0.6$ for iDose level 


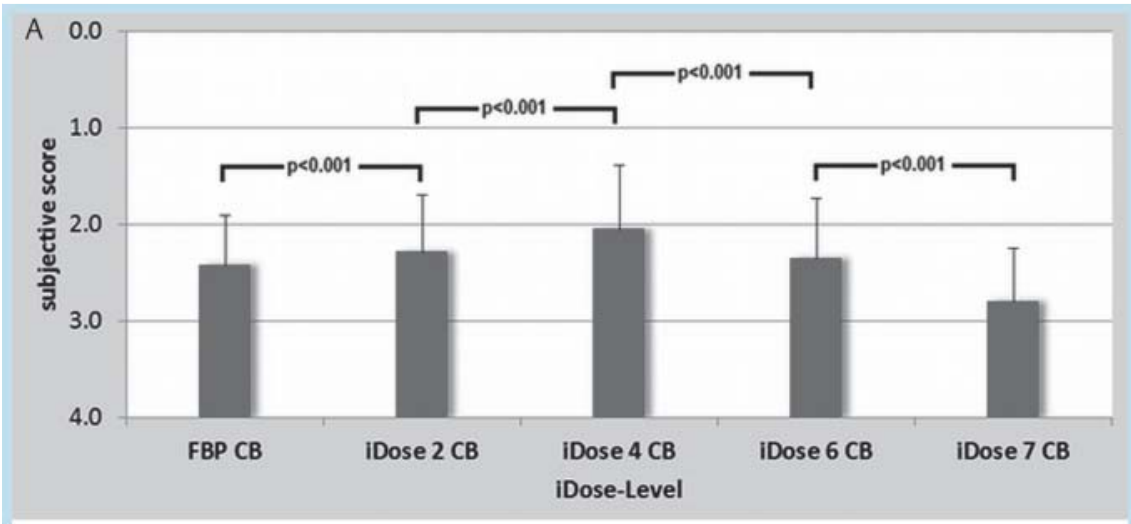

Fig. 4 Subjective image quality at FBP and increasing levels of IR for CB kernel (A) and XCB kernel (B), respectively. Best subjective scores were reached at a medium level of IR (iDose level 4) as compared to FBP and to the highest levels of IR.

Abb. 4 Subjektive Bildqualität bei FBP und ansteigenden Stufen der IR bei CB (A) und XCB (B). Beste subjektive Scores wurden bei mittleren IR-Stufe (iDose Stufe 4) erreicht.
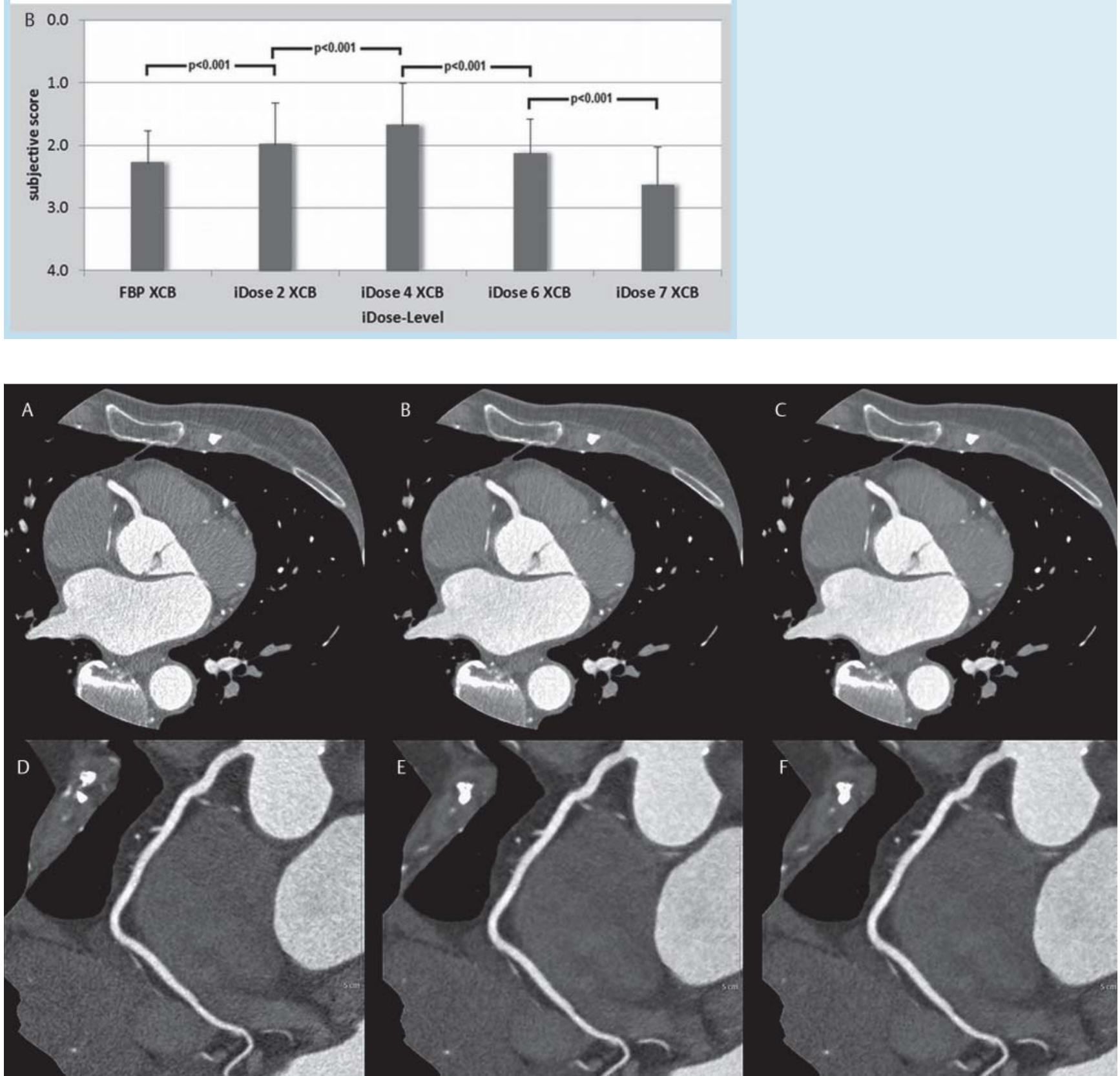

Fig. 5 Image examples. Axial (A-C) CT images of the aortic root and proximal RCA and curved MPRs of the RCA (D-F) in a 57-year-old male, demonstrating moderate subjective image quality (score 3 ) after FBP with kernel XCB (A, D), excellent image quality (score 1) at medium level (iDose 4 with $X C B)$ of IR (B, E) and good image quality (score 2 ) at highest level (iDose 7 with $X C B$ ) of IR $(C, F)$.
Abb. 5 Bildbeispiele. Axiale (A-C) CT-Bilder der Aortenwurzel und proximalen RCA sowie gekrümmte MPRs der RCA (D-F) bei einem 57-jährigen Patienten, die eine moderate subjektive Bildqualität (Score 3) bei FBP und XCB zeigen (A, D), eine exzellente Bildqualität (Score 1 ) bei mittlerer IRStufe (iDose 4 mit XCB) (B, E) und eine gute Bildqualität (Score 2) bei höchster Stufe (iDose 7 mit XCB) (C, F). 
6 and $1.8 \pm 0.6$ for iDose level 7, respectively, showing a significant improvement at all three levels $(\mathrm{p}<0.001)$ ( $\bullet$ Fig. 3 ).

The three radiologists demonstrated a substantial interobserver agreement regarding the subjective image quality (average Fleiss kappa with correction according to Brennan and Prediger of $0.74 \pm 0.12$ ).

A combined illustration of subjective and objective IQ with respect to the different IR levels is demonstrated in $\bullet$ Fig. 2. Examples of subjective image quality scoring (moderate, good, and excellent) at different IR levels are shown in $\bullet$ Fig. 5.

\section{Discussion}

\section{$\nabla$}

The present study demonstrates that increasing the levels of an advanced IR technique has the potential to essentially improve subjective and objective image quality in dose-optimized CCTA examinations in the clinical routine.

Radiation dose has raised increasing concern in modern radiology. Radiation exposure due to cardiac CT has been significantly reduced during the last years by implementation of various technical innovations [7 - 11]. However, dose reduction may compromise image quality, which remains the critical parameter in all "low-dose" examinations. The main drawback of FBP as a standard image reconstruction technique in CT is the rapidly increasing image noise relative to the effective radiation with the risk of non-diagnostic image quality due to photon starvation. A disproportional dose reduction resulting in non-diagnostic scans, however, must be avoided as the patient may be exposed to ionizing radiation without any benefit.

In this study, a CCTA protocol with optimized dose-related parameters was employed. The estimated mean effective dose (according to ICRP 60) of $1.7 \pm 0.7 \mathrm{mSv}$ was in the same range as in previous studies [11]. Using the high-pitch mode on a dualsource CT unit, the mean effective dose for small and averagesized can even be reduced below $1 \mathrm{mSv}[23,24]$.

All examinations of our study were fully diagnostic, even when conventional FBP was used for image reconstruction. When the advanced IR technique was applied, however, the objective and subjective image quality was significantly improved. The CNR was almost doubled at the highest level of IR when compared to FBP in our measurements.

Many studies have been published within the last years reporting on noise reduction and improvement of low-contrast image quality due to IR in CT examinations of the abdomen [13], the thorax $[14,15]$ and the heart [25 - 27]. In recent studies by Utsunomiya et al. and Laqmani et al., a progressive improvement of the CNR and subjective image quality in cardiac CT and lowdose chest CT was reported when using different levels of IR compared to FBP $[28,29]$.

Regarding objective image quality (noise, CNR), these results were quite concordant with the present study as the best CNR and the most effective noise reduction were observed at the highest level of IR (iDose level 7). Although a slight decrease in contrast between coronaries and adjacent epicardial fatty tissue was noted with increasing IR levels, the corresponding reduction in noise by far outweighs this effect, which is only observed in measurements using ROIs of very small size. Advanced IR algorithms enable a progressive separation of image information and noise. Due to the involvement of the raw data space in the reconstruction process, those IR techniques are not only capable of reducing image noise (domain of image space) but can also re- duce and prevent image artifacts (domain of raw data space) to further improve image quality.

In contrast to other studies, a discrepancy between the subjective and objective image quality at increasing levels of IR was found in this study. While the highest CNR values were measured at the highest level of IR, the best subjective image quality was found at a medium level of IR (iDose level 4). In this setting, noise-related artifacts were substantially reduced. In contrast to the highest levels of IR, however, the authentic CT image appearance, which the readers were used to from daily practice, was preserved at a low and medium level of IR. In a previous study using less advanced IR algorithms, differences in image appearance due to higher levels of IR have already been discussed [30]. Compared to FBP, CT images reconstructed using iterative algorithms may appear more "artificial" or "plastic" mainly due to a general smoothing effect and a loss of granular image appearance. This is true particularly for CT images acquired with a lower dose subjectively assigning radiation saving parameters to a grainier image appearance. Singh et al. describe a blotchy pixelated image impression when reporting about abdominal CT images using an adaptive IR technique [13]. The results of our systematic comparison of different levels of IR reflect the daily experience when dealing with IR. The image appearance of IR is different from images processed by FBP which radiologists are used to. However, it has to be emphasized that the image quality scores reported here represent the highly subjective image impression of the three readers in this study and may be different when other radiologists score the images. When using advanced hybrid IR, noise texture and spatial resolution were reported as constant by Utsunomiya et al., although slight differences between image texture between FBP and IR were described [28]. In our study, differences in the subjective image quality between the medium and high level of IR were found to be significant in favor of a medium level of IR leading to an improvement of almost $25 \%$. As a possible reason for the modified non-classic image appearance after IR, changes in the noise power spectrum (NPS) due to an alteration in raw data computing are discussed in the literature [31]. Particularly at lower frequencies, IR may influence noise characteristics. Whether this is the only key to explain the differences in subjective image impression has to be further clarified. Results for the "multi-resolution" feature described above, which modifies the NPS to preserve a typical CT image appearance, support this hypothesis as "multi-resolution" led to further improvement of the subjective IQ in higher levels of IR. However, this applies only in combination with the sharper XCB kernel, indicating that different forms of IR have to be used with particular respect to the characteristics of the reconstruction kernel in order to keep subjective image quality at an ideal level taking the requirements of the radiological task into account.

Regarding the two reconstruction kernels CB and XCB applied in this study, results for objective and subjective image quality differed slightly but significantly. Whereas the CNR of images processed with the CB kernel exceeded the CNR values of images generated with the edge-enhanced XCB kernels, the application of XCB led to better subjective image scores. The XCB kernel is used in order to produce more edge and vessel wall enhancement than can be achieved with the CB kernel. This not only compensates the increased noise from the XCB kernel but results in a better overall subjective image quality already with FBP. Thus, IR in combination with an edge-enhancing reconstruction algorithm (i.e. XCB) and "multi-resolution" represents an ideal combination, as high spatial resolution can be combined with a reduction 
in image noise while preserving an easy-to-adapt image appearance.

The results of our study are in good agreement with those from a similar study performed with the iDose prototype, which, however, was restricted to a maximum iDose level of 5 and the use of the XCB kernel only [32]. As a prototype that was not approved as a medical device was used at the point in time when this study was conducted, only the influence of increasing IR levels on image quality at a constant dose could be investigated. Although the results indicate that the improvements in image quality could be used for dose reductions at constant subjective image quality, it is not possible to state how large this reduction could be. However, as iDose has become a regular medical device in the meantime, this could be evaluated in an additional study.

Our study has some limitations. Firstly, as a retrospective analysis of clinical data was performed, a systematic bias cannot be totally excluded. Secondly, a systematic comparison of CCTA and invasive coronary angiography as a reference standard has not been performed in this study, so that a possible influence of CCTA with different IR levels on diagnostic accuracy could not be analyzed. Furthermore, only 11 of 30 patients were included for subjective image analysis, although this number is similar to comparable previously published series and these datasets represent the spectrum of cardiac patients in the clinical routine (normal coronaries, stenosis, stents and bypass grafts were included). Moreover, the results of subjective IQ analysis were very stable and a substantial change was not to be expected by expanding the analysis to all datasets. The CCTA protocol was not completely identical in all patients as the flow rate and amount of contrast as well as the z-coverage were adjusted individually in every patient. The study population itself was not homogeneous regarding BMI, heart rate, and thoracic diameters but reflects consecutive patients in the clinical routine. On the other hand, FBP and all levels of IR were performed on the same datasets. Radiation doses were calculated and not measured directly in this study. As the IR system of only one manufacturer was used in this study, the results cannot be automatically transferred to other IR systems of other manufacturers.

In conclusion, objective image quality progressively improves with increasing level of IR in dose-optimized CCTA with the best CNR values at the highest level of IR. The best subjective image quality, however, is achieved at medium levels of IR due to reduced artifacts and a preserved "classic" image appearance. The use of edge-enhancing reconstruction algorithms combined with a medium level of iterative reconstruction in combination with the NPS preserving "multi-resolution" feature proved to result in superior image quality in low-dose CCTA suggesting application in the clinical routine.

\section{Clinical relevance}

$\nabla$

Advanced iterative reconstruction techniques offer different increasing levels of IR. In low-dose CCTA, a medium level of iterative reconstruction combined with an edge-enhancing kernel leads to optimal subjective image quality suggesting application in clinical practice. Higher levels of IR further increase the objective image quality (CNR) but may affect the "classic" image appearance. Here, a combination with a "multi-resolution" feature can help to preserve the NPS.

\section{References}

1 Maruyama T, Yoshizumi T, Tamura R et al. Comparison of eight- versus 16-slice multidetector-row computed tomography for visibility and image quality of coronary segments. Am J Cardiol 2004; 94: 15391543

2 Ferencik M, Nomura CH, Maurovich-Horvat P et al. Quantitative parameters of image quality in 64-slice computed tomography angiography of the coronary arteries. Eur J Radiol 2006; 57: 373-379

3 Mowatt G, Cook JA, Hillis GS et al. 64-Slice computed tomography angiography in the diagnosis and assessment of coronary artery disease: systematic review and meta-analysis. Heart 2008; 94: 1386-1393

4 Fang XM, Chen HW, Hu XY et al. Dual-source CT coronary angiography without heart rate or rhythm control in comparison with conventional coronary angiography. Int J Cardiovasc Imaging 2010; 26: 323 - 331

5 Hausleiter J, Meyer T, Hermann F et al. Estimated radiation dose associated with cardiac CT angiography. JAMA 2009; 301: 500-507

6 Einstein AJ, Henzlova MJ, Rajagopalan S. Estimating risk of cancer associated with radiation exposure from 64-slice computed tomography coronary angiography. JAMA 2007; 298: 317-323

7 Feuchtner GM, Jodocy D, Klauser A et al. Radiation dose reduction by using $100-\mathrm{kV}$ tube voltage in cardiac 64-slice computed tomography: A comparative study. Eur J Radiol 2009; 75: e51 - e56

8 Hausleiter J, Meyer T, Hadamitzky $M$ et al. Radiation dose estimates from cardiac multislice computed tomography in daily practice: impact of different scanning protocols on effective dose estimates. Circulation 2006; 113: 1305-1310

9 Shuman WP, Branch KR, May JM et al. Prospective versus retrospective ECG gating for 64-detector CT of the coronary arteries: comparison of image quality and patient radiation dose. Radiology 2008; 248: 431 437

10 Stolzmann P, Leschka S, Scheffel $H$ et al. Dual-source CT in step-andshoot mode: noninvasive coronary angiography with low radiation dose. Radiology 2008; 249: 71-80

11 Klass $O$, Jeltsch M, Feuerlein S et al. Prospectively gated axial CT coronary angiography: preliminary experiences with a novel low-dose technique. Eur Radiol 2009; 19: 829-836

12 Ambrose J, Hounsfield G. Computerized transverse axial tomography. Br J Radiol 1973; 46: 148-149

13 Singh S, Kalra MK, Hsieh J et al. Abdominal CT: comparison of adaptive statistical iterative and filtered back projection reconstruction techniques. Radiology 2010; 257: 373 - 383 Epub 2010 Sep 9

14 Pontana F, Pagniez J, Flohr T et al. Chest computed tomography using iterative reconstruction vs. filtered back projection (Part 1): Evaluation of image noise reduction in 32 patients. Eur Radiol 2011; 21: 627-635 Epub 2010 Nov 5

15 Pontana F, Duhamel A, Pagniez J et al. Chest computed tomography using iterative reconstruction vs filtered back projection (Part 2): image quality of low-dose CT examinations in 80 patients. Eur Radiol 2011; 21: 636-643 Epub 2010 Nov 16

16 Lee SJ, Park SH, Kim AY et al. A prospective comparison of standarddose $\mathrm{CT}$ enterography and 50\% reduced-dose CT enterography with and without noise reduction for evaluating Crohn disease. Am J Roentgenol $2011 ; 197$ : 50-57

17 Kröpil P, Lanzman RS, Walther C et al. [Dose reduction and image quality in MDCT of the upper abdomen: potential of an adaptive post-processing filter]. Fortschr Röntgenstr 2010; 182: 248-253

18 Kröpil $P$, Cohnen $M$, Andersen $K$ et al. Image quality in multidetector CT of paranasal sinuses: potential of dose reduction using an adaptive post-processing filter. Fortschr Röntgenstr 2010; 182: 973-978

19 Mehta D, Bayraktar Dhanantwari. Effect of iterative reconstruction technique on image texture. Insights into Imaging 2011; 2: C-1938 C-2011. DOI: $10.1594 /$ ecr2011/C-1938

20 Ferencik M, Moselewski F, Ropers D et al. Quantitative parameters of image quality in multidetector spiral computed tomographic coronary imaging with submillimeter collimation. Am J Cardiol 2003; 92: $1257-1262$

21 Achenbach S, Giesler T, Ropers D et al. Comparison of image quality in contrast-enhanced coronary-artery visualization by electron beam tomography and retrospectively electrocardiogram-gated multislice spiral computed tomography. Invest Radiol 2003; 38: 119-128

22 Mayo JR, Aldrich J, Muller NL. Radiation exposure at chest CT: a statement of the Fleischner society. Radiology 2003; 228: 15-21

23 Achenbach S, Marwan M, Ropers D et al. Coronary computed tomography angiography with a consistent dose below $1 \mathrm{mSv}$ using prospec- 
tively electrocardiogram-triggered high-pitch spiral acquisition. Eur Heart J 2010; 31: 340 - 346

24 Kröpil P, Rojas CA, Ghoshhajra B et al. Prospectively ECG-triggered high-pitch spiral acquisition for cardiac CT angiography in routine clinical practice: initial results. J Thorac Imaging 2012 May 27: 194 - 201

25 Moscariello A, Takx RA, Schoepf UJ et al. Coronary CT angiography: image quality, diagnostic accuracy, and potential for radiation dose reduction using a novel iterative image reconstruction technique-comparison with traditional filtered back projection. Eur Radiol 2011; 21: $2130-2138$

26 Oda S, Utsunomiya D, Funama Y et al. A hybrid iterative reconstruction algorithm that improves the image quality of low-tube-voltage coronary CT angiography. Am J Roentgenol 2012; 198: 1126 - 1131

27 Renker M, Ramachandra A, Schoepf UJ et al. Iterative image reconstruction techniques: Applications for cardiacCT. J Cardiovasc Comput Tomogr 2011; 5: 225-230

28 Utsunomiya D, Weigold WG, Weissman G et al. Effect of hybrid iterative reconstruction technique on quantitative and qualitative image analy- sis at 256-slice prospective gating cardiac CT. Eur Radiol 2012; 22: $1287-1294$

29 Laqmani A, Buhk JH, Henes FO et al. Impact of a 4th Generation Iterative Reconstruction Technique on Image Quality in Low-Dose Computed Tomography of the Chest in Immunocompromised Patients. Fortschr Röntgenstr 2013; 185: 749-757

30 Leipsic J, Nguyen G, Brown J et al. A prospective evaluation of dose reduction and image quality in chest CT using adaptive statistical iterative reconstruction. Am J Roentgenol 2010; 195: 1095 - 1099

31 Marin D, Nelson RC, Schindera ST et al. Low-tube-voltage, high-tubecurrent multidetector abdominal CT: improved image quality and decreased radiation dose with adaptive statistical iterative reconstruction algorithm-initial clinical experience. Radiology 2010; 256: $450-459$

32 Hosch D, Stiller $W$, Mueller $D$ et al. Reduction of radiation exposure and improvement of image quality with BMI-adapted prospective cardiac computed tomography and iterative reconstruction. Eur J Radiol 2012; 81: $3568-3576$ 